Revue d'histoire de l'Amérique française

REVUE D.HISTOIRE DE L'AMÉRIQUE FRANÇAISE

\title{
La division sexuelle du travail et la modernisation de l'agriculture à travers la presse agricole, 1840-1900
}

\section{Martine Tremblay}

Volume 47, numéro 2, automne 1993

URI : https://id.erudit.org/iderudit/305218ar

DOI : https://doi.org/10.7202/305218ar

Aller au sommaire du numéro

Éditeur(s)

Institut d'histoire de l'Amérique française

ISSN

0035-2357 (imprimé)

1492-1383 (numérique)

Découvrir la revue

Citer cet article

Tremblay, M. (1993). La division sexuelle du travail et la modernisation de l'agriculture à travers la presse agricole, 1840-1900. Revue d'histoire de l'Amérique française, 47(2), 221-244. https://doi.org/10.7202/305218ar
Résumé de l'article

Éclairer la question du travail des femmes dans l'agriculture au XIX ${ }^{\mathrm{e}}$ siècle n'est pas une mince tâche car ces dernières n'ont à peu près pas laissé de traces de leurs activités quotidiennes. Cependant, certains éducateurs, journalistes et agronomes, dans leur volonté de diffuser leur vision de la modernisation de l'agriculture, ont traité de l'organisation du travail à la ferme. Quelle part font-ils aux femmes dans les tâches et les responsabilités associées à la pratique agricole? Comment ces élites, qui tentent de soumettre l'agriculture à de nouveaux impératifs, voient-elles le " progrès " pour les femmes?

À la fin du XIX ${ }^{\mathrm{e}}$ siècle, l'espace des femmes tel que présenté par les journaux d'agriculture s'est rétréci. Plus qu'un effet de la commercialisation croissante des produits de la ferme, l'affaiblissement du leadership des femmes dans certaines productions agricoles, comme celle du beurre, semble lié principalement à leur statut dans la société traditionnelle.
Tous droits réservés @ Institut d'histoire de l'Amérique française, 1993

Ce document est protégé par la loi sur le droit d'auteur. L'utilisation des services d'Érudit (y compris la reproduction) est assujettie à sa politique d'utilisation que vous pouvez consulter en ligne.

https://apropos.erudit.org/fr/usagers/politique-dutilisation/ 


\title{
LA DIVISION SEXUELLE DU TRAVAIL ET LA MODERNISATION DE L'AGRICULTURE À TRAVERS LA PRESSE AGRICOLE, 1840-1900
}

\author{
MARTINE TREMBLAY \\ Centre interuniversitaire d'études québécoises \\ Université du Québec à Trois-Rivières
}

\section{RÉSUMÉ}

Éclairer la question du travail des femmes dans l'agriculture au XIX ${ }^{\mathrm{e}}$ siècle n'est pas une mince tâche car ces dernières n'ont à peu près pas laissé de traces de leurs activités quotidiennes. Cependant, certains éducateurs, journalistes et agronomes, dans leur volonté de diffuser leur vision de la modernisation de l'agriculture, ont traité de l'organisation du travail à la ferme. Quelle part font-ils aux femmes dans les tâches et les responsabilités associées à la pratique agricole? Comment ces élites, qui tentent de soumettre l'agriculture à de nouveaux impératifs, voient-elles le «progrès» pour les femmes?

À la fin du $\mathrm{XIX}^{\mathrm{e}}$ siècle, l'espace des femmes tel que présenté par les journaux d'agriculture s'est rétréci. Plus qu'un effet de la commercialisation croissante des produits de la ferme, l'affaiblissement du leadership des femmes dans certaines productions agricoles, comme celle du beurre, semble lié principalement à leur statut dans la société traditionnelle.

\section{ABSTRACT}

Shedding light on women's work in agriculture in the $19^{\text {th }}$ century is not easy because women left almost no records of their daily activities. However, educators, journalists and agronomists discussed the organization of labour on the farm within their campaign to modernize agriculture. How did this reform-minded elite evaluate women's tasks and responsibilities and what role dit it see for them in its redefinition of agriculture?

In the end of the $19^{\text {th }}$ century, according to descriptions in agricultural journals, women's participation was reduced. More than a consequence of the growing commercialization of farm products, the diminishing importance of women in some types of production, such as butter, seems to be mainly linked to their status in traditional society.

1. Ce texte est inspiré de mon mémoire de maîtrise déposé à l'Université du Québec à Trois-Rivières en 1987. Je suis très reconnaissante à René Hardy qui a lu et commenté les différentes versions de cet article. Merci également à Jean Roy et Normand Séguin qui ont bien voulu me faire leurs commentaires.

RHAF, vol. 47, $\mathrm{n}^{\circ}$ 2, automne 1993 
La question du travail des femmes dans l'agriculture a donné lieu à de nombreuses publications dont un courant important constate le confinement progressif, au $\mathrm{XX}^{\mathrm{e}}$ siècle, des agricultrices dans les tâches de moindre importance, alors que la gestion de la ferme et le partage des tâches semblaient plus égalitaires au siècle dernier ${ }^{2}$. C'est une position endossée par les auteures de l'Histoire des femmes au Québec qui ajoutent, en s'inspirant des grands courants historiographiques, que l'industrialisation et la spécialisation agricole ont joué un rôle majeur dans le retrait des femmes de la production agricole ${ }^{3}$. La production manufacturière du tissu et ensuite des vêtements, puis la production laitière pour le marché auraient provoqué le retrait des femmes des activités dites productives en faveur des activités de consommation. Les hommes, seuls responsables des productions destinées à la vente, auraient accaparé du même coup le pouvoir économique et exerceraient plus que jamais leur autorité sur les autres membres de la famille. Il est toutefois difficile de vérifier cette assertion puisque les paysannes québécoises ont laissé peu de traces de leurs activités quotidiennes, ce qui explique la quasi-absence des travaux sur ce sujet.

Je propose ici l'étude des journaux et des traités d'agriculture, en dépit des limites que pose cette documentation, comme moyen d'appréhender le travail des hommes et des femmes vivant de l'agriculture au siècle dernier. Ce type de documents a été exploité avec profit par Sally McMurry, qui le considère, avec d'autres sources imprimées, plus intéressant que les recensements pour éclairer le travail des femmes ${ }^{4}$. Martine Segalen a aussi largement utilisé une source

2. Les principaux travaux publiés sur le travail des femmes dans l'agriculture au XIX $\mathrm{X}^{\mathrm{e}}$ siècle sont les suivants: Martine Segalen, Mari et femme dans la société paysanne (Paris, Flammarion, 1980), 211 p.; Marjorie Griffin Cohen, Women's Work, Markets, and Economic Development in Nineteenth-Century Ontario (Toronto, University of Toronto Press, 1988), 258 p.; «The Decline of Women in Canadian Dairying», Histoire Sociale/Social History, 17,34 (1984): 307-334; Joan M. Jensen, «Butter Making and Economic Development in Mid-Atlantic America from 1750 to 1850», Signs: Journal of Women in Culture and Society, 13,4 (1988): 813-829; With These Hands, Women Working on the Land (Old Westbury/New York, The Feminist Press/The McGraw-Hill Book Company, 1981); Sally McMurry, «Women's Work in Agriculture: Divergent Trends in England and America, 1800 to 1930», Comparative Studies in Society and History, 34,2 (avril 1992): 248-270. Pour une revue des thèmes et un bilan historiographique sur les femmes et l'agriculture au $\mathrm{XX}^{\mathrm{e}}$ siècle: Barbara J. Cooper, «Farm Women: Some Contemporary Themes», Labour/Le Travail, 24 (automne 1989): 167-180; «Femme et terre», numéro spécial de Pénélope, 7 (automne 1982); Rose-Marie Lagrave, «Bilan critique des recherches sur les agricultrices en France», Études rurales, 92 (octobre-décembre 1983): 9-40; «Women in Agriculture and Rural Society/Les femmes dans la production agricole et la société rurale», numéro spécial de Resources for Feminist Research/Documentation sur la recherche féministe (RFR/DRF), 11,1 (mars 1982).

3. Micheline Dumont, Michèle Jean, Yolande Lavigne et Jennifer Stoddart, L'histoire des femmes au Québec depuis quatre siècles (Montréal, Quinze, 1982), 186-187.

4. Sally McMurry, «Women's Work in Agriculture...», op. cit., 250. 
difficile à traiter, les proverbes, qui renvoie à la norme et même aux formes les plus figées de cette norme afin d'analyser les rapports étroits qui unissent le mari, la femme et la terre ${ }^{5}$. Je crois que les journaux, même s'ils ne nous parlent pas des accommodements que trouvaient chaque jour les paysans et les paysannes, nous renseignent abondamment sur la norme et son évolution au cours du XIX ${ }^{\mathrm{e}}$ siècle.

Les journalistes et les éducateurs considèrent-ils le travail des femmes et des hommes de la même manière? Leurs écrits nous permettent-ils d'affirmer que la modernisation est la cause principale du confinement des femmes dans les tâches agricoles subalternes? L'analyse des journaux et des traités devrait nous éclairer sur la perception qu'ont les rédacteurs des relations entre hommes et femmes dans une agriculture orientée vers la satisfaction des besoins de la famille. Peut-être y décèlerons-nous des rapports de dépendance préexistants à la transformation de la société. Mais surtout, il faut comprendre comment certaines élites entrevoient la réorganisation du travail pour satisfaire à la nécessaire modernisation de l'agriculture.

\section{LES JOURNAUX ET LES TRAITÉS D'AGRICULTURE}

La demande croissante des marchés urbains au cours du XIX siècle amène les paysans situés près des villes à restructurer progressivement leur production. Pendant ce temps, certaines élites, bouleversées par l'exode massif des paysans vers les États-Unis, cherchent les moyens d'accélérer la modernisation de l'agriculture afin de rentabiliser et de rendre plus attrayant le travail de la terre. Leurs efforts sont appuyés par un courant important qui favorise la modernisation de la production agricole à travers l'Occident; le développement des connaissances et l'amélioration des techniques, de même que la mise au point d'un outillage plus perfectionné viennent convaincre ces élites du caractère inéluctable de la transformation. Elles tenteront d'organiser la transmission de nouvelles connaissances par le biais de conférences, de visites d'agronomes, de fermes modèles, d'écoles d'agriculture $^{6}$. Le journal et le traité d'agriculture font partie de ces moyens utilisés pour inciter les paysans au changement.

Un peu moins d'une dizaine de journaux et près d'une vingtaine de traités d'agriculture forment le corpus de cette étude; nous en tracerons donc un portrait d'ensemble. Les traités ont été publiés en français au Québec (Bas-Canada) et ne sont ni des brochures, ni des

5. Martine Segalen, Mari et femme...

6. Bruno Jean, «Idéologie et professionnalisation: le cas des agronomes», Recherches sociographiques, 19,2 (mai-août 1978): 251-260. 
traités spécialisés sur l'élevage. Nous avons aussi choisi les journaux en fonction de critères de langue et de lieu d'édition. Enfin, nous avons tenté de réunir tous les journaux soutenus ou publiés par le gouvernement, la Société et la Chambre d'agriculture du Bas-Canada et le Conseil d'agriculture de la province de Québec.

Deux journaux méritent cependant une brève présentation en raison de leur plus grande stabilité dans le temps et de leur diffusion. La Gazette des Campagnes, journal agricole et catholique bimensuel puis hebdomadaire, est publiée de 1861 à 1895 presque sans interruption ${ }^{7}$. L'éditeur de la Gazette, situé à l'École d'agriculture de SainteAnne-de-la-Pocatière, reçoit de cette institution les fonds nécessaires au maintien de son entreprise en même temps que l'appui des élites cléricales pour le recrutement des abonnés. L'autre publication agricole d'importance au Québec est le Journal d'agriculture fondé par l'agronome E.-A. Barnard en 1876. Prise en charge par le ministère de l'Agriculture en 1877 , cette publication est distribuée à tous les membres des Cercles et des Sociétés d'agriculture: ces derniers doivent prélever le montant de l'abonnement à même leurs cotisations, ce qui assure au journal une large diffusion. Le tirage du Journal d'agriculture a quadruplé en vingt ans et dépasse, à la fin du siècle, les 40000 exemplaires $^{8}$.

Les sections de livres et surtout les articles de journaux abordant la division sexuelle du travail sont nombreux, parfois écrits pour le paysan, mais la plupart adressés explicitement aux femmes. Ces textes traitent à profusion de la femme idéale, dont la seule préoccupation est de faire le bonheur de sa famille en développant ses qualités d'ordre, d'économie, de piété, de discrétion. Les écrivains et les rédacteurs décrivent aussi très longuement et avec une grande précision la division du travail entre hommes et femmes. Ils dissèquent les tâches quotidiennes, critiquent les méthodes en usage, proposent de nouvelles manières de faire, suggèrent même une réorganisation du travail.

L'utilisation de cette source comporte toutefois ses limites; la presse agricole reflète une réalité sociale considérée normale, en même temps qu'elle propose, comme média d'information et d'éducation, de nouvelles normes visant l'amélioration de la qualité des produits agricoles, de la productivité et des conditions de vie et de

7. La publication est interrompue de novembre 1867 à avril 1868 en raison de difficultés financières.

8. Dix mille cinq cents exemplaires en 1877,20000 en 1893 et 46000 en 1898 (A. Beaulieu et J. Hamelin, La presse québécoise des origines à nos jours, 2: 1860-1879, 250). M.-A. Perron estime le tirage de la Gazette des Campagnes entre 2 et 3000 exemplaires (M.-A. Perron, Un grand éducateur agricole, E.-A. Barnard, 1835-1898, 318). 
travail. Cette volonté de modeler les pratiques entraîne le gommage des différences régionales et des décalages chronologiques. Cette source ne permet pas non plus de cerner avec précision l'impact de telle ou telle spécialisation ou d'une adaptation plus ou moins rapide des nouvelles techniques de production; il est encore moins possible d'entrevoir les différences dans l'organisation du travail qui résultent de la taille et de l'évolution du cycle de la famille, de même que de traditions familiales originales.

Les journaux d'agriculture posent un problème spécifique. Plusieurs des articles ont d'abord été publiés dans des journaux agricoles canadiens-anglais, américains et français; certains recoupements le révèlent. Il arrive aussi que les rédacteurs mentionnent la provenance de leur texte. Devrait-on exclure ces influences étrangères, si seulement il était possible de le faire? Je ne le crois pas car les rédacteurs qui puisaient dans les journaux étrangers considéraient certainement ces idées acceptables pour leurs lecteurs, ou du moins ils croyaient en leur valeur pédagogique. De plus, ce travail de repiquage donne à penser que les similitudes étaient nombreuses entre la vie quotidienne des agriculteurs d'ici, ceux des États-Unis et ceux de l'Europe occidentale en cette fin du XIX ${ }^{\mathrm{e}}$ siècle.

Un autre type de problème relève cette fois de l'analyse des traités; ces derniers laissent percevoir l'évolution des techniques de culture et d'élevage mais ils traitent rarement de la division des tâches parmi les membres de la famille. Ici point d'accommodement, point de discussion sur les avantages et les inconvénients des changements proposés. Malgré la sécheresse de leurs propos, ces livres bénéficient d'une bonne diffusion: certains d'entre eux sont écrits ou traduits à la demande du gouvernement et souvent utilisés comme manuels selon les directives du ministère de l'Instruction publique. Enfin, plusieurs de ces traités sont distribués en prix par les maîtres et les inspecteurs d'écoles.

Ces difficultés ne sont pas insurmontables et sont amplement compensées par l'avantage d'ajouter quelques éléments à notre connaissance des rapports sociaux fondés sur le genre ${ }^{9}$. Ces textes constituent le seul matériau dont nous disposons actuellement pour traiter de la division sexuelle du travail au sein de familles vivant d'agriculture au XIX ${ }^{\mathrm{e}}$ siècle.

9. Pierre Bourdieu, «La domination masculine», Actes de la recherche en sciences sociales, 84 (septembre 1990): 2-31 ; Nadia Fahmy-Eid, «Histoire, objectivité et scientificité. Jalons pour une reprise du débat épistémologique», Histoire sociale/Social History, 24,47 (mai 1991): 9-34; E. E. Maccoby, «Le sexe, catégorie sociale», Actes de la recherche en sciences sociales, 83 (juin 1990): 16-26; Joan Scott, «Genre: une catégorie utile d'analyse historique», Les cahiers du GRIF, numéro spécial «Le genre de l'histoire», 37 (printemps 1988): 125-153. 


\section{LE TRAVAIL DES FEMMES ET DES HOMMES}

La division du travail au sein de la famille est manifeste: tous les individus ne sont pas interchangeables dans toutes les activités. Certaines activités sont toujours exécutées par les femmes, d'autres sont réservées aux hommes, d'autres encore sont partagées. La ferme est en fait divisée en deux espaces qui s'interpénètrent dans certaines circonstances ${ }^{10}$ : l'espace féminin recouvre la maison et son environnement immédiat, l'espace masculin est constitué par les champs ${ }^{11}$.

La presse agricole associe étroitement les femmes au foyer, à la maison. Le soin et l'éducation des enfants, la préparation des repas, la confection et l'entretien des vêtements accaparent l'attention des femmes et exigent une surveillance constante ${ }^{12}$. Cet espace est tout de même assez vaste et n'est pas restreint aux activités ménagères. Il s'étend d'abord à l'entretien du jardin-potager. Responsables non seulement de la préparation des repas mais aussi des réserves pour l'hiver, elles planifient la production de fruits et de légumes ${ }^{13}$. Cette activité, même si elle est qualifiée de délassement et de distraction, est éminemment utile à la famille. Chargées de veiller à la bonne santé et au bien-être de tous, les femmes trouvent au jardin une variété d'aliments et une source d'économie ${ }^{14}$, tout en initiant les filles et les jeunes garçons à la pratique agricole ${ }^{15}$. Enfin, cette activité peut devenir lucrative lorsque la proximité des marchés urbains permet d'y vendre les primeurs ${ }^{16}$. Un verger, ou du moins quelques arbres fruitiers, et un rucher côtoient souvent le potager. Les femmes, encore là, observent, prodiguent leurs soins, et bien sûr recueillent les produits destinés à la consommation de la famille et à la vente ${ }^{17}$.

La basse-cour est aussi un espace féminin qui s'inscrit dans le prolongement du potager et de la cuisine. Les restes de table et les

10. Je reprends le concept d'espace tel que l'a proposé Lucienne A. Roubin dans «Espace masculin, espace féminin en communauté provençale», Annales ESC, 25,2 (mars-avril 1970): 537-560.

11. Louis Gossin, «L'agriculture et la famille», Gazette des campagnes, 43 (31 mai 1883): 344 .

12. «La science du ménage», Gazette des campagnes, 21 (11 mai 1877): 165. 329.

13. Mayre, «Le potager de la ferme», Gazette des campagnes, 41 (24 juillet 1873): 328-

14. «Avantage d'un jardin potager», Gazette des campagnes, 7 (20 avril 1893): 54.

15. «Causerie agricole: de la culture potagère», Gazette des campagnes, 36 (11 mars 1880): 284; «Du jardin potager», L'Agriculteur, journal officiel de la Chambre d'agriculture du Bas-Canada (juillet 1862): 260. $99-101$

16. «Causerie agricole: le jardin potager», Gazette des campagnes, 13 (28 mars 1878):

17. Abbé L. Provancher, Le verger, le potager et le parterre dans la province de Québec (Québec, C. Darveau, 1885), 168. 
pelures sont apprêtés et mélangés pour être distribués aux volailles ${ }^{18}$. Cet élevage est confié aux femmes parce qu'il requiert des soins quotidiens, «de la patience et de l'attention, et en même temps de la bonté et de la gentillesse, ce qui n'est pas toujours l'apanage du sexe laid ${ }^{19}$ ». Douceur et gentillesse des femmes, ou disponibilité face à un élevage exigeant? Les oiseaux de la basse-cour envahissent les champs et pillent le grain s'ils ne sont pas constamment surveillés ${ }^{20}$. Le poulailler doit être propre, chaud, bien aéré et l'eau doit être renouvelée quotidiennement. La surveillance constante dont il est l'objet permet de découvrir rapidement les maladies susceptibles de détruire l'élevage, de connaître les meilleurs éléments et de destiner les mauvais sujets à la casserole ${ }^{21}$. La basse-cour nécessite beaucoup de travail mais offre cependant des produits qui varient le menu et peuvent être vendus au marchand ${ }^{22}$.

L'espace féminin s'étend jusqu'à l'étable. L'élevage des porcs et des vaches, comme celui de la volaille, exige une attention soutenue ${ }^{23}$. La traite des vaches, par exemple, doit être exécutée ponctuellement, et la litière renouvelée à tous les jours ${ }^{24}$. Les femmes doivent leur procurer ce qu'il y a de mieux, veiller à ce qu'elles ne soient pas rudoyées. Les rédacteurs de journaux agricoles leur enjoignent de nettoyer l'étable, de laver les vaches et surtout le pis des vaches avant de les traire ${ }^{25}$. Il appartient aux femmes de connaître les bêtes, particulièrement les veaux, de savoir lesquelles devront être vendues ou abattues. De l'étable à la laiterie, les femmes conduisent les opérations, exécutent les tâches qui requièrent du doigté, surveillent de près les enfants ou les employés ${ }^{26}$. Elles font le fromage consommé à la ferme mais aussi le beurre, dont une grande partie de la production est vendue. Avec le lait de beurre elles nourrissent les porcs dont elles assurent aussi l'entretien ${ }^{27}$.

18. A. G. Gilbert, «Soin des volailles en hiver», Journal d'agriculture illustré (novembre 1893): 214 .

19. «La femme et le poulailler», Gazette des campagnes, 4 (16 janvier 1890): 28.

20. F. M. F. Ossaye, Les veillées canadiennes (Québec, Augustin Côté et cie, 1852), 133.

21. «Élevage et consommation des volailles», Gazette des campagnes, 1 (5 février 1892):

7; «Traitement des volailles en hiver, Gazette des campagnes, 3 (4 janvier 1877): 21-22;

«Constipation et diarrhée des volailles», Gazette des campagnes, 20 (11 mars 1875): 158-159.

22. «Éducation des volailles», Revue agricole (janvier 1863): 165.

23. Joigneaux, «Conseils à la jeune fermière», Gazette des campagnes, 12 (21 mars 1878): 94.

24. «Causerie agricole», Gazette des campagnes, 50 (23 janvier 1879): 387-389.

25. Annette de Beaupré, "L'ordre et la propreté», Journal d'agriculture illustré (décembre 1896): 117.

26. «Causerie agricole», Gazette des campagnes, 51 (6 février 1879): 395.

27. Joigneaux, "Conseils à la jeune fermière», Gazette des campagnes, 12 (21 mars 1878): 94. 
Les revenus que tirent les femmes des productions qu'elles dirigent et exécutent leur appartiennent; il semble même que leur mari ignore la valeur totale annuelle de ces ventes. Les femmes vendent les œufs, les volailles, le beurre, les légumes, les fruits, le miel et les fleurs pour acheter du tissu, des vêtements, des objets manufacturés de toutes sortes. Elles prennent seules les décisions qui concernent leurs responsabilités et dépensent le produit de leur travail sans en rendre compte à leur mari. Plusieurs rédacteurs reprochent d'ailleurs aux femmes de ne pas tenir un budget, ce qui les laisse dans l'ignorance de leurs revenus et de leurs dépenses ${ }^{28}$.

Les femmes devraient non seulement tenir un budget mais tenir aussi les livres de la ferme ${ }^{29}$. Madame Robinet n'ose revendiquer la tenue de la comptabilité de l'exploitation entière pour les femmes, mais elle croit qu'il serait avantageux pour les hommes de leur confier cette tâche.

Il serait juste et raisonnable aussi, écrit-elle, que les autres dépenses de l'exploitation fussent soumises à l'examen de la femme, qui pourrait faire aussi à leur sujet de judicieuses remarques. Les femmes, en général, ont un esprit de détail que n'ont pas les hommes, et, comme dans une exploitation agricole les détails sont immenses et se reproduisent dans toutes les opérations, une femme est très apte à donner un bon avis ${ }^{30}$.

Le changement proposé ne vise pas un partage de la gestion mais la tenue d'une comptabilité. La tenue de livres est conçue comme une tâche subalterne, importante certes, mais qui grugerait inutilement le temps précieux des hommes. Aussi, convient-elle davantage aux femmes et particulièrement aux jeunes filles ${ }^{31}$. Le budget familial dressé par Gauldrée-Boilleau est révélateur de la valeur différentielle du temps selon le sexe et l'âge: alors que l'évaluation du salaire de l'homme varie de 2,50 francs ${ }^{32}$ (pour les travaux des champs) à 0,75 franc par jour (pour le soin des bêtes à cornes), celui de la femme et de ses deux premières filles est généralement de 0,50 franc sauf pour la confection des vêtements qui vaut 1,00 franc par jour. Quant à la 413.

28. Madame Robinet, «Comptabilité», Gazette des campagnes, 52 (10 octobre 1872):

29. «Causerie agricole: ce que coûte la production d'un minot de blé», Gazette des campagnes, 33 (11 juin 1874): 257.

30. Madame Robinet, «Économie domestique: dépenses du ménage», Gazette des campagnes, 52 (10 octobre 1872): 413.

31. "Causerie agricole: ce que coûte la production d'un minot de blé», Gazette des campagnes, 33 (11 juin 1874): 257.

32. Il utilise le système monétaire français parce que cette monographie est destinée avant tout à un public universitaire français. 
fille cadette, qui contribue au travail agricole et domestique, son travail vaut généralement moins que celui de sa mère et de ses sœurs, à l'exception de la confection des vêtements ${ }^{33}$.

Pendant ce temps, les hommes sont occupés aux champs. Ils planifient les travaux de la grande culture, travaillent seuls ou avec leurs fils, quelquefois avec des employés. Ils décident d'investir dans l'achat d'équipement ou d'entreprendre des travaux d'amélioration et d'agrandissement ${ }^{34}$. Les hommes semblent exercer le plus grand pouvoir économique au sein de la famille malgré la latitude que détiennent les femmes grâce à leur production. Ces revenus, provenant de la vente de beurre et de quelques douzaines d'œufs, de quelques paniers de fruits et de légumes, sont considérés comme marginaux par les hommes qui contrôlent les revenus de la grande culture. Ils laissent leur femme régler de cette façon une partie de la note chez le marchand sans chercher à comptabiliser ces rentrées d'argent occasionnelles ${ }^{35}$. Le mari est d'ailleurs obligé de contribuer aux dépenses de la famille avec les revenus de ses transactions ${ }^{36}$.

Ce découpage de l'espace n'est pas exclusif. Hommes et femmes le transgressent de manière occasionnelle ou régulière, soit pour offrir leur aide, soit pour accomplir des tâches qui sont traditionnellement réservées à l'un ou l'autre sexe. Les rédacteurs des journaux agricoles, de même que Léon Gérin, considèrent que certaines tâches de l'espace masculin, comme les travaux de fenaison, de ramassage, de javelage, de sarclage, de repiquage, appartiennent aux femmes et aux enfants. Les observations de Gauldrée-Boilleau, par contre, en donnent une autre image: l'implication des femmes est si peu importante dans les travaux des champs qu'il n'en tient pas compte dans son évaluation des ressources et des dépenses de la famille ${ }^{37}$. Cette situation s'explique sans doute par l'emploi de travailleurs salariés qui obtiennent environ trente journées par année ${ }^{38}$. Les monographies de Gérin et Gauldrée-Boilleau et les articles de journaux donnent à penser que la rareté de la main-d'œuvre ou l'impossibilité pour la famille d'y avoir recours, de même que l'absence de grands enfants, obligent les femmes à participer davantage aux travaux des champs ${ }^{39}$.

33. Gauldrée-Boilleau, «Paysan de Saint-Irénée de Charlevoix en 1861 et 1862», 47.

34. «Comptabilité agricole», Gazette des campagnes, 3 (17 janvier 1878): 23.

35. «Importance de la volaille pour les cultivateurs», Journal d'agriculture et transactions de la Société d'agriculture du Bas-Canada, 6,2 (février 1853): 76; «Avantage d'un jardin potagen», Gazette des campagnes, 7 (20 avril 1893): 54.

36. Madame Robinet, «Economie domestique: dépenses du ménage», Gazette des campagnes, 52 (10 octobre 1872): 413.

37. Gauldrée-Boilleau, «Paysan de Saint-Irénée...», op. cit., 46.

38. Ibid., 32. 262.

39. «Le rôle de le femme dans la vie rurale», Gazette des campagnes, 33 (4 juin 1886): 
Les travaux des femmes aux champs sont généralement des gestes répétitifs supervisés par les hommes. Elles participent à l'ensemencement des plantes racines, comme la betterave, elles sarclent, récoltent, nettoient ${ }^{40}$. Le ramassage de la récolte des pommes de terre est réservé aux femmes et aux enfants: tandis que les hommes arrachent les plants à la main ou à la charrue, les femmes, aidées des enfants, placent les tubercules dans des paniers qu'elles vident ensuite dans des sacs ou des tombereaux ${ }^{41}$. Elles ont aussi leurs tâches dans la récolte des céréales et du foin. La faucille est maniée par les femmes, et elle permet aux hommes d'utiliser indistinctement tous les bras disponibles $^{42}$. Le ramassage et la mise en gerbe sont une tâche presque exclusivement féminine ${ }^{43}$. Un temps pluvieux n'entraîne pas leur retrait de ces rudes travaux des champs, au contraire, car cette situation occasionne l'augmentation du nombre d'opérations. Lorsque les moissons doivent être faites malgré le mauvais temps, les gerbes sont alors rassemblées en petites meules provisoires dressées au champ: quatre ou cinq femmes apportent les gerbes à un homme qui construit la meule ${ }^{44}$. Les femmes s'occupent aussi de la récolte des tiges de blé d'Inde qu'elles coupent à la faucille, ramassent, lient et disposent en meules ${ }^{45}$. La récolte du foin requiert également son contingent de femmes; il faut compter habituellement quatre femmes pour un faucheur ${ }^{46}$. Les femmes sont chargées, souvent avec les enfants, du fanage, mais elles sont écartées du déchargement sur les aires d'engrangement parce que c'est un travail pour lequel «des hommes et même des hommes vigoureux conviennent mieux que des femmes ${ }^{47}$ ». Les femmes participent donc très activement aux travaux des champs mais n'y exercent jamais de pouvoir décisionnel, sauf en l'absence prolongée de leur mari ${ }^{48}$. Ce dernier planifie le travail, décide ce qui

40. M. de Dombasle, «De la betterave, sa culture, sa récolte et sa conservation», Journal d'agriculture et transactions de la Société d'agriculture du Bas-Canada, 1,6 (juin 1848): 162163.

41. «Quelle est la meilleure manière d'arracher les patates?», Revue agricole (octobre 1862): 27; Antoine de Roville, «De la récolte des racines», L'Agriculteur, journal officiel de la Chambre d'agriculture du Bas-Canada (septembre 1858): 32-33; A. P. C. R. Landry, Traité populaire d'agriculture (Montréal, Imprimerie canadienne, 1878), 260.

42. Ibid., 144.

43. «Figure 3 - Manière de faire les liens et les moyettes», Journal d'agriculture illustré (août 1894): 143.

44. M. De Dombasle, «Moisson dans les saisons pluvieuses», Journal d'agriculture et transactions de la Société d'agriculture du Bas-Canada, 1,1: 29.

45. Correspondance de Charles Hugues, «Récolte de fourrage», Journal d'agriculture et transactions de la Société d'agriculture du Bas-Canada, 5,1 (janvier 1852): 3.

46. «Fanage des foins», Revue agricole (juillet 1863): 263.

47. Ibid.

48. Madame Robinet, «Économie domestique», Gazette des campagnes, 50 (26 septembre 1872): 397. 
est plus urgent, requiert la présence des femmes pour exécuter certaines tâches. Le travail des femmes est subordonné aux activités de la grande culture; en période d'activités intenses, elles vont délaisser la cuisine, la laiterie et le potager et minimiser les soins à donner aux animaux.

Les hommes pénètrent eux aussi l'espace féminin pour y exécuter certaines tâches. Leur aide est nécessaire dans des travaux de jardinage: le bêchage, le transport des engrais, l'ameublissement de la terre avant l'hiver sont réservés aux hommes ${ }^{49}$. Ils doivent encore se prêter de bonne grâce aux requêtes de leur femme pour réparer les meubles, la maison ou les bâtiments, ou pour nettoyer la cour. Mais plusieurs femmes exécutent elles-mêmes ces petits travaux de menuiserie et d'entretien ${ }^{50}$. Enfin, les hommes s'occupent de certains animaux: les chevaux ou les gros bestiaux, qui les aident dans les travaux des champs, de même que les moutons ${ }^{51}$.

Les transgressions de l'espace féminin par les hommes semblent cependant moins nombreuses et moins encouragées que l'inverse. Il est plus vraisemblable de rencontrer une femme ayant pris la direction des travaux des champs en raison de l'absence, de la maladie ou du décès de son mari qu'un homme occupé aux soins de la basse-cour et du potager. Non seulement y a-t-il des tâches féminines aux champs, mais les femmes doivent être capables de remplacer leur mari en tout temps. Leur présence occasionnelle assure une surveillance que les hommes ne peuvent pas toujours exercer, particulièrement si la famille emploie des travailleurs ${ }^{52}$. Par contre, nulle part les rédacteurs de la presse agricole ne disent aux femmes qu'elles peuvent compter sur leur mari pour un coup de main occasionnel; même l'aide qu'ils doivent généralement apporter, comme le transport des engrais au potager ou les réparations à l'étable et à la maison, doit être demandée par les femmes avec tact et circonspection.

\section{LES SAVOIR-FAIRE DES FEMMES DISCRÉDITÉS}

La presse agricole reflète la pensée d'un groupe d'agronomes et d'éducateurs qui se sont donné la mission de diffuser les dernières

49. «Causerie agricole: de la culture potagère», Gazettte des campagnes, 36 (11 mars 1880): 284; «Du jardin potager», L'Agriculteur, journal officiel de la Chambre d'agriculture du Bas-Canada (juillet 1862): 260.

50. «Qu'est-ce qu'une bonne ménagère?», Journal d'agriculture illustré (octobre 1896): 77; «La bonne ménagère», Journal d'agriculture et d'horticulture (8 juin 1899): 548.

51. Joigneaux, «Conseils à la jeune fermière», Gazette des campagnes, 12 (21 mars 1878): 94.

52. «De l'éducation des fermières», Gazette des campagnes, 23 (15 octobre 1862): 182. 
découvertes dans le domaine de l'agronomie. Leurs discours, leurs écrits ou leurs idées sont repris par les rédacteurs des journaux agricoles, et ces propos dénoncent presque unanimement les pratiques traditionnelles des femmes pendant tout le demi-siècle. La remise en question des savoir-faire des femmes est totale, elle englobe toutes les facettes de leurs tâches et de leurs responsabilités. Je ne présenterai cependant que quelques exemples qui touchent deux sujets abondamment traités, la direction du poulailler et celle de la production laitière: ils suffiront à illustrer le mode d'intervention de cette élite.

Espaces traditionnellement réservés aux femmes, le poulailler, l'étable et la laiterie sont scrutés surtout par des hommes, qui reprochent aux femmes leurs coutumes anciennes, non fondées scientifiquement, et tentent de leur imposer de nouvelles pratiques. Ils décrivent parfois les usages les plus courants, en les marquant du sceau de la bêtise, avant d'entreprendre leur travail d'éducation. À compter des années 1880 , quelques-uns vont pousser un peu plus loin la dévaluation du travail des femmes en les accusant de négligence. Visaient-ils expressément l'exclusion des femmes de certaines productions agricoles? Je ne saurais le dire à la lumière de ces recherches. Mais une lecture attentive de ces écrits permet d'observer un groupe qui, dans sa volonté d'acculturer les masses populaires, élabore tout un discours justifiant le retrait des femmes de certaines activités, par exemple la confection du beurre.

L'accent mis ici sur le travail des femmes ne doit pas faire oublier que les hommes ont aussi été confrontés au changement dans leurs pratiques et leurs savoirs traditionnels ${ }^{53}$. L'abbé Provancher, discutant du jardinage, représente bien le point de vue de certaines élites sur les problèmes du monde rural. Il exprime son insatisfaction devant la tenue des jardins et assure que cette activité devrait nourrir la famille, payer tous les frais de culture et procurer un bénéfice qui pourrait être investi dans des travaux d'amélioration et d'agrandissement. Mais il ajoute: «Il en est malheureusement de la culture jardinière comme de notre grande culture, une routine aveugle, malentendue, irrationnelle très souvent, tient la place des vrais principes et des méthodes raisonnées et éprouvées. On ne lit pas $^{54}$ !» Pour l'homme comme pour la femme, l'observation des plus vieux, l'apprentissage auprès de la mère ne suffisent plus; il faut lire, recevoir un enseignement, se renseigner.

53. Le discrédit jeté sur les pratiques et les savoirs traditionnels ne caractérise pas les écrits des seuls éducateurs et agronomes du Québec. Une étude de l'acculturation des masses populaires sous l'effet de la modernisation en Occident reste à faire.

54. Abbé L. Provancher, Le verger, le potager..., 169. 
Les pratiques courantes, le savoir transmis de mère en fille, deviennent des préjugés et entraînent le mépris. Un rédacteur de L'agriculteur ridiculise le comportement de certaines femmes, encore incultes, avant d'étaler un savoir technique. Il écrit qu' «il ne faut pas, comme le font encore quelques ménagères, prendre les œufs, les plonger dans de l'eau froide ou dans de l'eau chaude, sous prétexte d'attendrir la coquille et de rendre l'éclosion plus facile. Ce ne sont là que des préjugés $(. . .)^{55} . »$ Un autre s'exclame: «Encore un préjugé! Le tonnerre tue dans leur coquille les poussins prêts à éclore! (...) Un peu de surveillance et de soin feraient meilleur effet que des morceaux de fer plus ou moins rouillés, placés en croix ou en nombre impair ${ }^{56} .{ }{ }^{2} \mathrm{La}$ diffusion de la pensée scientifique ne va pas sans heurter la culture populaire; ici la dérision sert de repoussoir aux idées et aux pratiques traditionnelles. Le même procédé est utilisé pour dénigrer le travail des femmes à la laiterie. Joigneaux trace un portrait railleur d'une femme barattant de la vieille crème:

Plus d'une fois, sans doute, tu as vu de pauvres ménagères se fatiguer, s'essouffler à battre de la crème sans réussir à faire prendre le beurre. Elles en accusent habituellement la mauvaise chance ou les sorciers (...). Le sorcier, c'est la vieille crème; le sorcier, c'est encore parfois la température ${ }^{57}$.

Parmi ces auteurs, plusieurs sont conscients de la vague qui les porte et perçoivent la cause du décalage entre la pratique quotidienne et leurs connaissances. Joigneaux représente bien cette tendance; il considère les femmes victimes d'un système d'enseignement d'où elles sont systématiquement exclues. Le mode de transmission de la science agronomique inverse les rôles, conduit à «l'aberrante» situation où les femmes responsables de telle ou telle production n'en connaissent pas les nouveaux développements. «Les ménagères qui s'entendent bien aux choses de la laiterie, écrit-il, ne sont pas communes. (...) Il y a toute une science là-dessous, science que l'on enseigne aux hommes, mais que l'on enseigne point aux femmes. C'est tourner le dos au sens commun $(. . .)^{58} . »$ Cela ne l'empêche pas, cependant, de ridiculiser certaines pratiques, comme l'exemple précédant l'a démontré, ou de dénoter l'absurdité des croyances. Par

55. «La poule cochinchinoise, et particulièrement de la production des œufs», L'Agriculteur, journal officiel de la Chambre d'agriculture du Bas-Canada (novembre 1861): 65.

56. Vortelier, «L'influence du tonnerre sur les couvées», Gazette des campagnes, 50 (17 juillet 1884): 399.

57. Joigneaux, «Conseils à la jeune fermière», Gazette des campagnes, 7 (28 novembre 1872): 55 .

58. Ibid. 
exemple, il explique que les femmes laissent vieillir la crème ou ajoutent au beurre des matières étrangères, comme du jus de carottes ou de fleurs de soucis, parce qu'elles accréditent l'idée que le bon beurre doit être jaune ${ }^{59}$.

Qu'ils raillent ou expliquent les méthodes en usage, les auteurs en viennent toujours à proposer de nouvelles façons de faire. Ils analysent le processus de fabrication du lait, puis celui du beurre, de la prairie jusqu'au seau qui recueille le lait, et de là au marché où est vendu le beurre. On dit aux femmes, par exemple, qu'elles ne traient pas les vaches assez longtemps et perdent en conséquence la portion la plus riche du lait ${ }^{60}$. Ailleurs, on dénonce les mauvais traitements subis par les vaches, à l'étable ou aux champs, assurant que ces comportements ont une influence néfaste sur leur rendement. Il ne suffit pas de nourrir les animaux et de changer leur litière; ils doivent être considérés comme des partenaires dans la production laitière.

Tu auras pour tes vaches, écrit Joigneaux, toutes sortes d'égards qu'on leur refuse généralement. À l'étable comme au pâturage, on les maltraite, on les frappe du fouet ou du bâton, on lance sur elles les chiens. (...) Tu traiteras ces animaux avec douceur et ne souffriras pas que d'autres les traitent durement ${ }^{61}$.

Enfin, on relie aussi le rendement à la qualité de la nourriture et de $1^{\prime} e^{6}{ }^{62}$. On incite les femmes à demander à leur mari de préparer de bonnes prairies et de bons fourrages ${ }^{63}$. On les encourage à procurer le plus tôt possible aux vaches de l'herbe tendre qui donne au lait une qualité supérieure, en expliquant que la déficience de l'alimentation à l'automne cause leur tarissement ${ }^{64}$. Enfin, on leur dit que la traite est une opération délicate qui ne peut être confiée à n'importe qui ${ }^{65}$.

59. Ibid.

60. William Evans, Traité théorique et pratique de l'agriculture (Montréal, Louis Perreault, 1836-1837), 299; «Importance d'un trayage bien exécuté», Gazette des campagnes, 47 (22 juin 1882): 373 .

61. Joigneaux, "Conseils à la jeune fermière», Gazette des campagnes, 13 (28 mars 1878): 94.

62. «Animaux de la ferme», Revue agricole (octobre 1862): 28; M. Robinet, «Économie domestique», Gazette des campagnes, 50 (26 septembre 1872): 397.

63. «Confection du beurre sur la ferme», Gazette des campagnes, 1 (5 février 1892): 45. «Altération et falsification du beurre», Gazette des campagnes, 43-44 (28 novembre 1878): 341-342. F.-X. Valade, Guide de l'instituteur (4éd., Montréal, J.-B. Rolland, 1856), 305.

64. «Le beurre de mai», Gazette des campagnes, 30 (8 juin 1876): 238. «De la perte due aux mauvais traitements des vaches à cette saison», Gazette des campagnes, 16 (16 novembre 1882): 126.

65. Annette de Beaupré, «L'ordre et la propreté», Journal d'agriculture illustré (décembre 1896): 117. 
Les rédacteurs expliquent ensuite l'importance de la régularité et de la précision à la laiterie. Ils suggèrent fortement l'utilisation du thermomètre ${ }^{66}$, ou de tel type de récipients plutôt que tel autre ${ }^{67}$. Ils recommandent la construction d'une glacière qui permettra de bien conserver le beurre jusqu'au moment de la vente sans devoir y ajouter une forte dose de sel ${ }^{68}$. Ils considèrent très important que la laiterie soit un endroit aéré, propre et blanchi à la chaux ${ }^{69}$, strictement réservé à la manipulation du lait ${ }^{70}$, et que l'extérieur de la laiterie soit aussi bien entretenu ${ }^{71}$. Les auteurs des articles et des manuels donnent aux femmes des règles, pas toujours concordantes, sur le temps de barattage $^{72}$, le lavage du beurre ${ }^{73}$, le temps que l'on peut faire attendre la crème avant de baratter ${ }^{74}$. Les contradictions ne favorisaient certainement pas un changement rapide des méthodes de travail. Les femmes continuaient probablement de se fier aux techniques traditionnelles de fabrication. L'auteur d'un manuel d'agriculture explique pourquoi les femmes doivent modifier leur manière de travailler: si les Canadiennes ne réussissent pas à faire d'aussi bon beurre que les Écossaises, écritil, c'est «seulement parce qu'elles n'expriment pas assez leur beurre pour en tirer tout le lait; et c'est si bien la seule raison, que les Américains achètent le beurre Canadien (sic) pour le manipuler de nouveau et le vendre ensuite chez eux comme le beurre de première qualité ${ }^{75}$ ». Fondée ou non, cette affirmation révèle la perception d'un important courant de pensée: les lacunes du travail des femmes fait perdre à

66. Joigneaux, «Conseils à la jeune fermière», Gazette des campagnes, 7 (28 novembre 1872): 55; «Conférences agricoles pour les femmes des cultivateurs», Journal d'agriculture illustré (août 1895): 36; «Beurre ayant une forte odeur», Gazette des campagnes, 23 (5 janvier 1882): 181-182.

67. «Densité du lait», L'Agriculteur, journal officiel de la Chambre d'agriculture du BasCanada (septembre 1862): 15. 398.

68. J. D. S., «Établissement des glacières», Gazette des campagnes, 50 (10 mars 1870):

69. L. Gallicher, «Du rôle des femmes en agriculture», Gazette des campagnes, 34 (6 juin 1872): 270.

70. «Confection du beurre sur la ferme», Gazette des campagnes, 1 (5 février 1892): 45; «Économie domestique», Gazette des campagnes, 8 (15 février 1864): 65; «Causerie agricole: la fabrication du beurre», Gazette des campagnes, 4 (23 août 1883): 28; «Le beurre», Gazette des campagnes, 9 ( 7 mai 1862): 72.

71. «Facilité du beurre à prendre un mauvais goût», Gazette des campagnes, 3 (17 janvier 1878): 22-23; «La propreté est le point essentiel d'une bonne laiterie», Gazette des campagnes, 30 (20 mai 1875): 237-238.

72. «Beurre ayant une odeur forte», Gazette des campagnes, 23 (5 janvier 1882): 181-

182; «Battage du beurre», Gazette des campagnes, 34 (29 mars 1883): 272-273.

73. «Fabrication et traitement du beurre», Gazette des campagnes, 35 (5 avril 1883): 280-

281; «Le lavage du beurre», Gazette des campagnes, 50 (19 juillet 1883): 400.

74. «La fabrication du beurre», Gazette des campagnes, 20 (10 mars 1887): 155.

75. Ossaye, Les veillées canadiennes, 122. 
l'agriculture québécoise non seulement sa renommée mais aussi ses profits.

Si certains des rédacteurs comprennent l'enjeu de leurs interventions, c'est-à-dire la transformation des pratiques et des croyances populaires, d'autres font des femmes les boucs émissaires des problèmes d'adaptation de l'agriculture. Ceux-ci leur reprochent de produire du beurre qui se vend mal parce qu'elles font preuve de négligence à l'étable et à la laiterie. Un auteur écrit que «le lait en général coule naturellement bon sous la main de la laitière; et c'est sa faute si elle n'en fait pas de bon beurre et de bon fromage ${ }^{76} \gg$. Un autre attire l'attention sur la traite: «On doit faire en sorte de ne jamais laisser tomber aucune saleté dans le seau qui sert à traire les vaches. Quelques ménagères donneront pour excuse de ce manque de précaution, que le lait doit passer au couloir ${ }^{77} . »$ Plus graves sont les accusations de dénaturation du beurre portées contre les femmes. Dans le but d'en augmenter la quantité, certaines laitières se livreraient à des altérations, en mêlant au produit des «pommes de terre, du suif, de la craie ou du sable». Elles placeraient, au centre du morceau, du beurre de qualité inférieure ou du fromage ${ }^{78}$. Quelques auteurs attribuent la baisse du prix du beurre à ces opérations qui donnent mauvaise réputation au beurre canadien ${ }^{79}$.

Il est difficile de croire que de telles pratiques étaient répandues. Les propos des rédacteurs agricoles, marqués par le discours «hygiéniste» à la mode, participent d'un mouvement dont le but est d'accélérer la transformation des pratiques agricoles, de répondre à la concurrence que subit le beurre canadien sur les marchés extérieurs en engageant les agriculteurs à se spécialiser dans la production laitière. Ces accusations de négligence et de malhonnêteté ont certainement pour effet de déconsidérer le travail des femmes dans l'opinion publique, de rendre le beurre domestique moins en demande sur les marchés intérieur et extérieur, favorisant en cela l'expansion des beurreries.

Les changements proposés par la presse agricole ne vont pas de soi, la répétition des mêmes recommandations et critiques sur plus d'un demi-siècle en est la preuve. Et ce genre de discours a été relevé

76. «Laiteries», Journal d'agriculture et transactions de la Société d'agriculture du BasCanada, 2,6 (juin 1849): 179.

77. "Causerie agricole. La fabrication du beurre», Gazette des campagnes, 4 (23 août 1883): 28.

78. «Altérations et falsification du beurre», Gazette des campagnes, 43-44 (28 novembre 1878): 342 .

79. Idem.; «Vaches laitières-beurre», Gazette des campagnes, $43-44$ (28 novembre 1878): 342. 
aussi en Ontario, aux États-Unis et en Grande-Bretagne ${ }^{80}$. Les méthodes de fabrication du beurre, par exemple, sont apprises avec la mère et répétées depuis très longtemps; en déroger implique un risque que les femmes ne semblent pas disposées à assumer. Malgré les moqueries et la profusion des explications scientifiques, les rédacteurs se butent à un mur d'indifférence, autant de la part des hommes que des femmes. Les changements auront tout de même lieu, et il en résultera un bouleversement de l'organisation du travail, une centralisation dans les mains des hommes du pouvoir décisionnel et financier au sein des familles qui continueront à vivre de l'agriculture, et à l'exclusion de milliers d'autres de la pratique agricole.

\section{MÉCANISATION ET COMMERCIALISATION: EXCLUSION DES FEMMES?}

Les changements proposés avant 1880 ne supposent pas une transformation radicale de l'organisation du travail à la ferme. Ils visent l'adaptation des savoir-faire aux nouvelles découvertes plutôt que la redistribution des tâches et des responsabilités au sein de la famille. Exceptionnellement en 1872, un article relie l'exode des travailleurs vers les villes, les transformations de la production et la participation des femmes aux activités de la grande culture. Son auteur, Gallicher, écrit que l'introduction de la culture des racines, le développement des méthodes d'élevage et les changements dans les méthodes de culture exigés par la pratique d'une agriculture «raisonnée ou scientifique» créent une grande demande de main-d'œuvre. Les femmes peuvent, grâce à la mécanisation, offrir leur aide aux travaux des champs.

L'émigration, écrit-il, nous enlève les plus vigoureux; l'attraction des villes, la domesticité dorée, les hauts salaires que leur offre l'industrie, nous privent des plus intelligents. - Il faut bien que les femmes de nos campagnes viennent à notre secours. Les machines, en supprimant la partie de nos travaux la plus dure et la plus difficile, leur ont ouvert l'accès de l'atelier agricole ${ }^{81}$.

Les femmes se voient intégrées à toutes les étapes des nouvelles productions à l'exception de la gestion. Les femmes sont des aides qui coopèrent au développement de l'agriculture, au moment où la main-

80. Marjorie Cohen Griffin, «The Decline of Women...», op. cit., 327; Joan M. Jensen, «Butter Making and Economic Development...», op. cit., 828; Sally McMurry, «Women's Work in Agriculture...», op. cit., 254.

81. L. Gallicher, «Du rôle des femmes en agriculture», Gazette des campagnes, 35 (13 juin 1872): 277. 
d'œuvre salariée fait défaut, sans toutefois remettre en cause l'exercice du pouvoir décisionnel par les hommes.

Il n'est d'ailleurs pas certain que la mécanisation a effectivement amené les femmes plus souvent aux champs. Un article publié en 1879 explique aux agriculteurs qu'il est préférable d'acheter un rateau à cheval et une machine à faner pour exécuter le fanage. Cet équipement procure «aux cultivateurs qui (l')adoptent l'économie de vingt faneuses pendant la fenaison ${ }^{82}$ ». Les orientations de la division sexuelle du travail au vingtième siècle nous portent à croire que s'il y a eu une plus grande contribution des femmes aux travaux des champs, cette participation n'a pas été reconnue. La mécanisation a peut-être intensifié le recours à la main-d'œuvre féminine, mais les conditions de travail de ces femmes se confondent désormais avec celles du travailleur agricole.

La production du lait et des œufs pour satisfaire les besoins du marché entraîne des changements décisifs dans l'organisation du travail. À la fin des années soixante-dix, dans un premier article qui traite de l'élevage des poules comme d'une spécialité, on s'adresse directement aux hommes: «Les profits immenses que les cultivateurs retirent par l'élevage des poules, vu le commerce considérable qui se fait par la vente des œufs, doit être pour les cultivateurs une raison d'attacher le plus grand soin à ce genre d'industrie ${ }^{83}$.» Sans contredit, on est ici persuadé que l'organisation de la production pour les marchés nécessite le leadership des hommes. Si les femmes continuent de nourrir les poules, elles ne doivent plus exercer la direction du poulailler.

La tendance à l'élargissement de l'espace masculin au détriment des responsabilités féminines s'amplifie dans les années quatre-vingt. Jusque-là, on réclamait des femmes l'amélioration de la qualité du beurre par la modification des procédés de fabrication et de l'outillage. La publication d'un premier article, en 1883, qui évoque l'idée de fabriquer le beurre à la beurrerie, est le prélude d'une nouvelle tendance. E.-A. Barnard, par exemple, conseille aux agriculteurs d'apporter le lait à une beurrerie-fromagerie plutôt que d'essayer de faire du beurre de première qualité à la ferme. Seul un spécialiste, soutient-il, peut fabriquer un excellent beurre ${ }^{84}$. Plusieurs articles publiés dans la Gazette des Campagnes étayent progressivement les

82. «La fenaison», Gazette des campagnes, 7 (24 juillet 1879): 53. 179.

83. «Causerie agricole: élevage des poules», Gazette des campagnes, 23 (1 juin 1877):

84. E.-A. Barnard, «Beurreries particulières», Journal d'agriculture illustré (février 1886): 32 . 
arguments en faveur d'un tel changement. On prétend d'une part que le beurre domestique est inférieur au beurre produit à la fabrique, soit par «ignorance des meilleurs procédés à employer pour la fabrication (...) ou négligence quant à ses différentes manipulations ${ }^{85}{ }_{\text {». }}$. D'autre part, on affirme la prédominance du beurre de fabrique à cause de l'expérience et de la disponibilité du fabricant et de l'outillage dont il dispose. Enfin, les femmes doivent souscrire à l'implantation des beurreries dans leur paroisse au nom du «progrès agricole» et de la percée des «marchés étrangers», parce qu'il n'y aura bientôt «de marché ouvert que pour le beurre fabriqué dans ces établissements ${ }^{86}$ ». En dépit de l'argumentation soutenue par la presse agricole, la qualité des produits de fabrique, beurre et fromage, laisse fortement à désirer si l'on en croit les propos de l'agronome Barnard et ceux d'un inspecteur du gouvernement rapportés dans l'article de Normand Perron $^{87}$. Les rédacteurs justifient aussi le changement par l'allègement des tâches qu'il procure. Les heures que consacraient les femmes à la fabrication du beurre peuvent ainsi être redistribuées à la faveur des autres responsabilités qui leur échoient ${ }^{88}$. La fabrique permet donc d'économiser le temps «car il n'est plus besoin, dès lors, dans chaque ménage, d'une personne consacrant plusieurs heures à la manipulation du lait ${ }^{89}$ ». Selon S. M. Barré, «les occupations ordinaires d'une fermière sont trop nombreuses et trop variées pour lui permettre d'accorder à la confection du beurre, le temps, le soin et l'attention nécessaire ${ }^{90}$ ».

Dans la dernière décennie, le silence tombe sur les responsabilités agricoles des femmes dans la plupart des journaux et particulièrement dans le Journal d'agriculture illustré. Sans être plus nombreux, les écrits qu'on leur adresse sont plus visibles puisqu'ils sont souvent regroupés dans une section féminine. Mais on n'y trouve plus, désormais, d'articles se rapportant à l'élevage des poules ou des vaches. Ce sujet, qui relève maintenant de la grande culture, prend place dans les premières pages des journaux, tandis qu'on publie pour les femmes des chroniques de puériculture, de cuisine et de décoration intérieure.

85. «Beurre fabriqué à la beurrerie», Gazette des campagnes, 25 (24 janvier 1884): 199.

86. «La fabrication du fromage et les beurreries», Gazette des campagnes, 12 (18 octobre 1883): 94-95.

87. Normand Perron, «Genèse des activités laitières, 1850-1960", op. cit.

88. «Causerie agricole: étude sur le fonctionnement et l'organisation des fromageries et des beurreries», Gazette des campagnes, 24 (12 janvier 1882): 187-189; «La fabrication du fromage et les beurreries", Gazette des campagnes, 12 (18 octobre 1883): 94-95.

89. «Des avantages des beurreries et fromageries pour la prospérité de l'agriculture», Gazette des campagnes, 18 (23 février 1888): 144.

90. S. M. Barré, professeur, «Industrie laitière», Gazette des campagnes, 42 (22 mai 1884): 333-334. 
Le discours qui promeut l'abandon de la production domestique doit donc être rapproché d'un mouvement plus vaste valorisant le rôle de la mère. Ce mouvement d'idées augmente l'importance des responsabilités liées à l'éducation des enfants et repousse les femmes vers l'univers domestique ${ }^{91}$.

Les rédacteurs ont attiré notre attention sur les défauts de la production domestique, son inefficacité, sa faible productivité, sa qualité inégale d'une part, et d'autre part sur l'économie de temps et d'énergie pour justifier le transfert de la production de la maison à la fabrique. Mais l'analyse des facteurs qui ont provoqué ce changement devrait également tenir compte des valeurs culturelles qui ont encouragé l'abandon de la production domestique de beurre et de fromage. Comme l'a bien démontré McMurry, les fromagères de GrandeBretagne ont résisté beaucoup plus longtemps que les Américaines, et jusqu'à la crise de 1929, aux tentatives d'implantation des fromageries. Occupant une position centrale dans l'organisation du travail des petites exploitations du nord et de l'ouest de la Grande-Bretagne, la fabrication du fromage relevait d'une tradition ancienne, fortement associée aux femmes. Ces dernières ont su conserver jalousement les secrets du processus de fabrication, tandis que leurs maris ne voyaient pas l'utilité de payer un fabricant pour effectuer un travail assumé gratuitement par leurs filles et leurs femmes. Pendant que les Anglais défendaient la position des femmes dans cette production, aux ÉtatsUnis, les rédacteurs de la presse agricole invoquaient, comme ici, l'économie de temps et de travail pour justifier l'abandon de la production domestique ${ }^{92}$.

Les Québécoises ont-elles résisté aux transformations de l'organisation du travail? Il semble qu'elles ont refusé de modifier les procédés de fabrication domestique du beurre mais qu'elles ont accepté assez facilement le transfert de cette production de la maison à la fabrique, si l'on considère le rythme d'établissement des beurreries à partir des années $1880^{93}$. Que le changement ait eu lieu rapidement ne signifie pas que les femmes l'ont accepté avec joie, mais les traités et les journaux ne permettent pas de percevoir leur résistance. Il est également possible que certaines d'entre elles y aient vu une amélioration de leurs conditions de travail, puisque le barattage n'était pas

91. Ce mouvement est caractéristique du monde occidental. Voir, entre autres, Julie A. Matthaei, Histoire économique des femmes aux États-Unis (Lausanne, Suisse, L'Âge d'Homme, 1985), 412 p.; Yvonne Knibielher et Catherine Fouquet, Histoire des mères, du Moyen Âge à nos jours (Paris, Montalba, 1977), 359 p.; Louise Vandelac et al., Du travail et de l'amour. Les dessous de la production domestique (Montréal, Éditions Saint-Martin, 1985), 418 p.

92. Sally McMurry, «Women's Work in Agriculture...», op. cit., 248-270.

93. Normand Perron, «Genèse des activités laitières...», op. cit., 119. 
une mince tâche. L'univocité du discours de la presse agricole masque également les initiatives visant à offrir aux femmes un enseignement agricole: les écoles ménagères, dès la fin du $\mathrm{XIX}^{\mathrm{e}}$ siècle, puis les Cercles de fermières, plus tard au $\mathrm{XX}^{\mathrm{e}}$ siècle, étaient au départ des moyens mis en place par des femmes, religieuses et laïques appuyées par un groupe de l'élite, pour maintenir ou reprendre leur place dans la production agricole ${ }^{94}$. Cette question de la résistance des femmes, de même que l'étude des facteurs qui ont facilité la réorganisation du travail, devront être explorées avec d'autres sources qui rendraient compte de la diversité des expériences de modernisation.

\section{COMPLÉMENTARITÉ ET HIÉRARCHISATION DES ESPACES}

Les espaces féminin et masculin se complètent mais ne sont pas strictement fermés l'un à l'autre. Les échanges sont par contre inégaux. Si les hommes ne remplacent pratiquement jamais les femmes et les aident rarement, ces dernières traversent de manière régulière ou intermittente l'espace masculin. La norme laisse très peu de place aux hommes dans l'espace féminin, et même leur pouvoir sur les décisions et les revenus des femmes est minime. Martine Segalen a très bien démontré comment la complémentarité des espaces était au cœur de l'organisation du travail ${ }^{95}$. L'exploitation de la terre semble presque impossible à l'homme autant qu'à la femme seule. Cette interdépendance des hommes et des femmes est perceptible à travers la presse agricole, mais on y entrevoit aussi la hiérarchisation des espaces. En fait, les relations entre les sexes se rapprochent de ce qu'a vu Léon Gérin dans la famille Casaubon: la femme ne se considère pas l'inférieure de son mari, mais il est tout de même significatif que ce soit elle et non lui qui se tienne debout pour servir à table ${ }^{96}$. Les femmes sont maîtres à l'intérieur de leur domaine; elles vont cependant subordonner leurs activités à celles de leur mari. Elles se tiennent au courant des affaires de la ferme, donnent leur avis si celui-ci leur demande, exécute pour lui des tâches qu'il n'a pas le temps de faire. Les femmes sont disponibles. Sans aucun doute, les rédacteurs, de même que Léon Gérin et Gauldrée-Boilleau, accordent aux espaces féminin et masculin une importance d'inégale valeur. À la complémentarité des espaces doit donc s'ajouter leur hiérarchisation, avant même que la production soit orientée vers la satisfaction des besoins du marché.

94. Nicole Thivierge, Écoles ménagères et instituts familiaux: un modèle féminin traditionnel (Québec, Institut québécois de recherche sur la culture, 1982), 475 p.; Yolande Cohen, Femmes de parole. L'histoire des Cercles de fermières du Québec, 1915-1990 (Montréal, Le Jour, 1990), $320 \mathrm{p}$.

95. Mari et femme dans la société paysanne.

96. J.-C. Falardeau et al., Léon Gérin et l'habitant de Saint-Justin, 86. 
Il va sans dire que la modernisation de l'agriculture a eu un impact important sur l'organisation du travail au sein de la famille. Les rédacteurs annoncent un rétrécissement de cet espace féminin au profit de l'espace masculin, où se retrouvent concentrés les pouvoirs décisionnels et les responsabilités. Les femmes continuent certainement d'aider au soin et à la traite des vaches, à la manipulation du lait ou aux travaux des champs, mais leur leadership n'est plus reconnu. Premières artisanes de la commercialisation de certains produits de la ferme, comme le beurre, les œufs, les fruits et les légumes, les femmes se sont déchargées de leurs lourdes responsabilités en même temps qu'elles abandonnaient la reconnaissance sociale qui y était associée. Les rédacteurs des journaux d'agriculture leur ont demandé de le faire au nom de l'efficacité, du progrès, d'un allègement de leurs tâches. Mais ce discours justifiait un ensemble de règles qui en appelait aux valeurs et aux représentations. Avant même le début de la modernisation, certaines caractéristiques de l'organisation sociale prédisposaient soit à la marginalisation des activités féminines, soit à l'exclusion des femmes des activités devenues centrales dans l'exploitation familiale.

L'organisation du travail au sein de la famille agricole du XIX siècle reflète le statut socialement accordé à l'un et à l'autre sexe. Les femmes exercent des responsabilités essentielles à la survie de la famille, mais ce sont les hommes qui détiennent la plus grande autorité. En fait, l'interdépendance des membres de la famille dans une agriculture peu intégrée à la société marchande ne signifie pas égalité des sexes, encore moins des générations. Une fine ordonnance distingue les travaux les plus nobles des autres qui sont subalternes, et d'autres encore qui sont dédaignés, généralement confiés aux vieux et aux enfants. Les femmes et les hommes organisent et dirigent les activités à l'intérieur de leur propre espace mais ces derniers peuvent déstructurer la planification décidée par les femmes en invoquant l'urgence et la priorité de leurs travaux. Les espaces féminin et masculin sont complémentaires et hiérarchisés ${ }^{97}$. Non seulement l'espace est-il hiérarchisé, l'espace féminin étant subordonné à l'espace masculin, mais la valeur du temps est-elle différente selon l'âge et le sexe. Ainsi,

97. Cécile Dauphin, Arlette Farge et al., ont bien expliqué comment les concepts de complémentarité et de hiérarchisation pouvaient se soutenir mutuellement: «Si la complémentarité rend effectivement compte d'une réalité où l'association de la femme et de l'homme s'avère nécessaire, elle gomme le fait que la distribution des tâches possède malgré tout un pôle positif et un pôle négatif et contient en elle un système de valeur hiérarchique. (...) Ces recherches, et bien d'autres, suggèrent de prendre désormais en compte non seulement la division technique des tâches, mais les valeurs et les symboles qui leur sont attachés.» "Culture et pouvoir des femmes: essai d'historiographie», Annales ESC, 2 (mars-avril 1986): 271-293. 
le temps des hommes est trop précieux pour qu'ils s'occupent euxmêmes de l'élevage des veaux, d'un rucher et même de la comptabilité. Ce sont les femmes qui seront responsables de ces activités, mais elles-mêmes en confieront l'exécution aux enfants, et plus particulièrement aux jeunes filles, dont le temps est le moins précieux de la famille. Cette ordonnance explique pourquoi, par exemple, Célina Wolff, épouse de Louis Casaubon, s'occupe seule de la production du tabac pendant que les femmes célibataires, tantes et filles, traitent la paille dans le but de confectionner les chapeaux ${ }^{98}$. Chez la famille Gauthier, la mère exécute les travaux les plus durs mais dont la valeur est supérieure au filage et tricotage confiés aux filles ${ }^{99}$.

Les productions les plus prestigieuses sont réservées aux hommes. Il n'est pas indifférent que la grande culture, celle qui fonde le patrimoine, assure la prospérité à long terme et permet l'établissement de la descendance, soit un espace masculin. La production laitière, par exemple, parce qu'elle devient de plus en plus lucrative mais aussi parce qu'elle est valorisée par les autorités gouvernementales, l'élite et finalement l'ensemble de la société, attire les hommes, et ce mouvement repousse les femmes vers d'autres activités, marginales. Le faible statut de la production domestique du fromage dans l'ensemble de la production agricole en Angleterre a constitué un rempart contre la déféminisation, constate Sally McMurry. C'est pourquoi elle conclut qu'à l'analyse des facteurs économiques doit nécessairement s'ajouter celle des traits culturels ${ }^{100}$. Bien que Marjorie Griffin Cohen relie principalement le recul des femmes dans la production du fromage et du beurre à leur impossibilité d'accumuler du capital, elle insiste d'abord sur la valeur inégale du travail des hommes et des femmes dans la société pré-industrielle ${ }^{101}$. Partenaires dans l'exploitation de la terre, hommes et femmes n'exercent cependant pas les mêmes droits sur leur propriété ${ }^{102}$; ces dernières sont placées dans une position désavantageuse dans le processus d'accumulation du capital ce qui, à terme, entraînera leur évincement de la production manufacturière $^{103}$. Les Québécoises ont, elles aussi, perdu le contrôle de la

98. J.-C. Falardeau et al., Léon Gérin et l'habitant de Saint-Justin, 60 et 62.

99. Gauldrée-Boilleau, «Paysan de Saint-Irénée de Charlevoix en 1861 et 1862», 33.

100. S. McMurry, «Women's Work in Agriculture...», op. cit., 270.

101. M. G. Cohen, Women's Work in Agriculture...», op. cit., 6-8.

102. Pas plus que les Ontariennes, les Québécoises n'ont le droit de gérer leurs biens. L'évolution de leurs droits est bien expliquée par les auteures de Histoire des femmes au Québec..., 331ss. Même sous le régime matrimonial de la séparation, les femmes doivent obtenir l'autorisation de leur mari ou d'un juge pour administrer leurs biens, 339.

103. Fernand Ouellet affirme que M. G. Cohen n'amène aucune preuve du déclin des femmes dans la production laitière («La question sociale au Québec, 1880-1930: la condition féminine et le mouvement des femmes dans l'historiographie», Histoire sociale/Social History, 
fabrication du beurre à mesure que s'implantaient les beurreries et que croissait l'intérêt des hommes pour cette production.

\section{CONCLUSION}

La vision des rapports sociaux développée dans les journaux d'agriculture repose sur la dépendance des femmes à l'égard des hommes, tout en consacrant aussi l'interdépendance des différents membres de la famille. Le statut conféré aux femmes en tant que groupe, qui les associe aux tâches et aux responsabilités les moins prestigieuses avant le début de la modernisation, détermine leur marginalisation lorsque vient le temps de concevoir cette modernisation.

Le discours tenu dans ces journaux nous parle autant sinon davantage des valeurs partagées par l'élite que du travail des hommes et des femmes; d'autres études devront être menées avant que l'on ait un éclairage suffisant de la question. La modernisation de l'agriculture a-t-elle entraîné des modifications sensibles de l'organisation du travail? À quel moment, dès la fin du XIX $\mathrm{X}^{\mathrm{e}}$ siècle ou plutôt vers le milieu du $\mathrm{XX}^{\mathrm{e}}$ ? Est-ce que les femmes avaient accès aux nouveaux savoirs en dehors des articles lus dans les journaux d'agriculture? Le gouvernement semble avoir joué un rôle important dans la modernisation de l'agriculture au XIX ${ }^{\mathrm{e}}$ siècle, comme le souligne justement Marjorie Griffin Cohen, rôle qu'il faudrait revoir en se demandant s'il a pu défavoriser les femmes. Dans la première moitié du $X X X^{e}$ siècle, l'État québécois va encourager le maintien ou même le rétablissement de la production des femmes par le biais de subventions aux Cercles de fermières et d'un programme de formation professionnelle. Toutefois, il est significatif de constater l'absence de la production laitière sur la liste des activités auxquelles doivent s'adonner celles qui désirent devenir membres, liste établie par le ministère de l'Agriculture dans les statuts des Cercles ${ }^{104}$. Enfin, une meilleure connaissance des particularités de l'organisation du travail imposées par les grandes spécialisations nous permettrait de mieux cerner l'évolution de la place des femmes dans l'agriculture.

21,42 (novembre 1988): 319-345). Il appuie son argumentation sur la valeur différentielle de la production entre les campagnes francophones et les cantons. Il faudrait connaître la méthodologie employée avant de juger de la pertinence d'une telle objection. Il est encore prématuré de traiter de la spécialisation laitière, c'est pourquoi je parle ici d'une commercialisation croissante. Cependant, il ne fait pas de doute que le développement de l'industrie laitière ne suit pas le même mouvement au Québec et en Ontario; aussi faut-il en faire l'analyse à l'échelle du comté pour l'ensemble du Québec, ce qui constitue un projet de SOREP actuellement. Une critique du texte de F. Ouellet a été publiée sous la plume de Micheline Dumont: «L'histoire des femmes», Histoire sociale/Social History, 23,45 (mai 1990): 117-128.

104. Yolande Cohen, Femmes de parole. L'histoire des Cercles de fermières du Québec, 1915-1990 (Montréal, Le Jour, 1990), 28. 